\title{
La responsabilidad social y los principios de la formación ciudadana como elementos de incorporación en la educación
}

\author{
Social responsibility and principles of citizenship education as elements of \\ incorporation in education
}

Recibido junio 2019

Arbitrado julio 2019

Publicado septiembre 2019
Luis Guillermo Quintero Galbán

qluisguillermo@gmail.com

Código ORCID: 0000-0002-7819-8753

Universidad del Zulia, Venezuela

\section{Resumen}

La responsabilidad social está orientada en la educación a la institución como organización, los entes profesionales que en ella participan y la comunidad educativa en general. Para participar activa y responsablemente en las decisiones colectivas de manera democrática, resolviendo conflictos en forma pacífica y a su vez para respetar la diversidad humana, entre otros importantes factores a los que hace relación la ciudadanía actual. La investigación tuvo como objetivo fundamental develar la responsabilidad social y los principios de la formación ciudadana como elementos de incorporación en la educación. La metodología usada en el presente estudio fue descriptiva con un enfoque bibliográfico, utilizando un cuestionario que arrojó como resultado la pertinencia de intervenir en el proceso educativo, teniendo para ello que deben haber factores externos que refuercen los valores ya existentes y para eso se planteó en este trabajo la responsabilidad social y la formación ciudadana como elementos claves en este proceso.

\footnotetext{
Abstract

The main objective of the research is to unveil social responsibility and the principles of citizen education as elements of incorporation into education. The social responsibility is oriented in the education to the institution as an organization, the professional entities that participate in it and the educational community in general. To participate actively and responsibly in collective decisions in a democratic manner, resolving conflicts in a peaceful manner and in turn to respect human diversity, among other important factors to which current citizenship relates. The methodology used in this research was descriptive with a bibliographic approach, using a questionnaire that shows as results that it is pertinent to intervene in the educational process, having for it, that there must be external factors that reinforce the existing values and for that it is proposed This work is social responsibility and citizen training as key elements in this process.
}

Palabras clave:

Responsabilidad social; formación ciudadana; educación

\section{Keywords:}

Social responsibility; citizen training; education 


\section{INTRODUCCIÓN}

L a educación ciudadana representa la oportunidad para formar valores de buena convivencia y tolerancia, orientada hacia la construcción de la paz en nuestra sociedad. Para ello esta debe partir de la convicción de que el mundo actual evoluciona y cambia. Educar y aprender constantemente es una necesidad individual y colectiva.

Hoy en día se vive en un mundo poco preocupado por los valores forjados desde el inicio de nuestra historia y la formación de la nación que sustentan nuestras bases sociales. Es por ello que nace esta inquietud, la cual está dirigida a proporcionar políticas educativas en las cuales podamos estimular la educación con el fin de que logren una responsabilidad social y una formación ciudadana.

Para Márquez (2007) al ser la educación un proceso permanente, se traza el propósito de transformar a los individuos para tomar un rol dinámico dentro del contexto de la sociedad y expresar un sentido de solidaridad social. Este valor cívico planteado desde la triada republicana bajo los lineamientos de Fraternidad, deben ser impulsados mediante un esfuerzo continuo orientado desde la educación formal y familiar.

Este propósito resulta una meta por alcanzar en nuestra sociedad. De modo que a los educadores se les convoca de forma inminente y además urgente a asumir este compromiso. Hoy es necesario entender el protagonismo que se tiene en el desarrollo de la nación, este debe partir de un compromiso individual con el sentido de pertenencia que de todo ciudadano. Dicha obligación debe partir de una educación familiar que encuentre un respaldo en el aula de clase. Por todo ello, se hace necesario reforzar la pedagogía ciudadana en todas las unidades curriculares afines vinculadas a la convivencia de los seres humanos.

Resulta importante para nuestra sociedad recuperar la fuerza del estímulo ético y moral en los hogares así como en las escuelas. Es preciso educar, sin distinción de géneros y a todos los niveles, futuros ciudadanos y ciudadanas con sentido de vida, convencidos del compromiso social. Tarea que corresponde desde la etapa paterno familiar con apoyo del educador sembrar conocimiento conceptual y actitudinal para el logro de una mentalidad ciudadana que apoye y promueva los procesos de paz y convivencia. 
En Venezuela existen diversos problemas por los cuales es necesaria la intervención inmediata de medios que ayuden a mejorar nuestro sistema de vida, en un mundo en donde el ser humano se aleja cada vez más de una ciudadanía inclusiva para pertenecer a una ciudadanía exclusiva.

El hombre, el ciudadano, el individuo, hoy en día se enfrenta a problemas muy notorios como la inseguridad, la corrupción, la desigualdad social, la pobreza. Para luchar contra ello la solución va más allá de una decisión política, para combatirlo lo que se requiere es, prácticamente, una acción de efecto moral y educativo a largo plazo, que involucre a todos los sectores y expresiones de su sociedad.

\section{La educación y lo social}

Se parte de la concepción del Estado Docente sustentado en la afirmación de que todo sistema educativo obedece a una concepción acerca del ciudadano o ciudadana que desea formar y tiene como base las aspiraciones y expectativas de la sociedad en la cual está inserta. El paradigma educativo que se construye en el país tiene como centro al ser humano como ser social, capaz de responder y participar activamente en la transformación de la sociedad en la que vive y se desenvuelve para desarrollar la pluralidad de pensamientos entre la familia, escuela y comunidad (Educere, 2007, pp.156-167).

Luego de una serie de reflexiones se considera que la pertinencia social en la educación es aquella que obliga al sistema educativo a responder ante la sociedad sobre lo que las instituciones a nivel nacional generan como producto de las enseñanzas impartidas para los alumnos, está vinculada con la responsabilidad y permite, de este modo, romper la posibilidad de un cerco de aislamiento que es incompatible con la misma tradición latinoamericanista de un sistema educativo que debe estar en armonía con las necesidades de la sociedad civil.

En Venezuela, haciendo referencia al tema de la responsabilidad que los gerentes educativos deben tener en sus instituciones educativas, hemos escuchado mucho en nuestras aulas la frase del Libertador "Moral y luces son nuestras primeras necesidades", entendiendo la educación como parte vital para la construcción de un algo o de un todo. Al hablar de ciudadanía en nuestro país, la polarización política existente puede dificultar el ejercicio de planes que permitan por medio de la formación ciudadana que el gerente 
guíe a su institución hacia la responsabilidad, el compromiso que debe tener la misma.

Sin embargo, siguiendo el ejemplo de otros países como el antes mencionado se debe partir de la idea de que este nuevo paradigma educativo va mucho más allá de intereses particulares y de doctrinas que se enmarcan en un campo cien por ciento humanista. En el mundo actual de modo más general, se habla de crisis de identidad, de fe y de epistemología.

Arana y Batista (2009) manifiestan que de identidad por la ausencia de un sentido claro de pertenencia y por la carencia de proyectos comunes unificadores; de fe, por la incapacidad de creer en algo, por la imposibilidad de cambio y la falta de confianza en el futuro; y epistemológica por la supremacía del conocimiento y la razón, que se expresa en una racionalidad instrumentaladministrativa-gerencial, capaz de aplastar lo afectivo y sentimental.

En tal sentido el hombre, el ciudadano, el individuo, hoy en día se enfrenta a problemas muy notorios como la inseguridad, la corrupción, la desigualdad social, la pobreza. Para luchar contra ellos la solución va más allá de una decisión política. Se requiere para combatirlo de una acción social de efecto moral y educativo que involucre a todos los sectores de la sociedad. Aunado a esto, los trabajadores se encuentran con problemáticas gerenciales que pueden perjudicar el buen desarrollo de la instrucción ciudadana que cada estudiante necesita.

Las reflexiones anteriores permiten criticar al ciudadano docente como un ente que debe ir llevado de la mano con la interrelación personal. Es importante acotar que en los centros educativos existen varios aspectos que deben tomarse en cuenta para el estudio del medio laboral y las consecuencias que dicho funcionamiento está trayendo a relucir en esta oportunidad. El mundo actual está enmarcado por un concepto de globalización, transformación en donde los educadores y líderes gerenciales deben ser partícipes de este proceso.

Por lo tanto, haciendo reflexión sobre factores, personas y entes que inciden en la formación de nuevos ciudadanos, surgió la siguiente pregunta en el proceso investigativo: ¿Qué hace el docente para crear un sentido de pertenencia por nuestras instituciones? Si se caracterizan las instituciones venezolanas se revela que, en estos últimos tiempos históricos, se ha disminuido la inversión del Estado en infraestructura, servicios, alimentos, papelerías. Por otra parte, la 
falta de comunicación en los hogares debido a la pérdida de valores ciudadanos hacen que nos enfrentemos a unos estudiantes indisciplinados sin conciencia ciudadana, esto trae a relucir diversos elementos que pueden estar afectando la carencia de valores en los ciudadanos.

En este mismo sentido, los entes gubernamentales también entran en estos posibles indicadores de problemas debido a que el docente no es bien remunerado y esto acarrea descontento, tanto en las instituciones públicas como en las privadas, se ve reflejado en nuestro entorno que existen docentes sin vocación que fue su última opción ejercer dicha profesión y es por esto que no se involucran en este tipo de conflictividades.

En torno a la meta que se planteó en esta investigación las características primordiales que se observaron son una cultura de violencia en las aulas, unos futuros ciudadanos poco comprometidos con los procesos educativos, sociales y políticos del país. Una Venezuela plena de coyunturas de gran impacto, pues, especialmente se destacó en este estudio que en las organizaciones educativas se observó despreocupación por los asuntos comunitarios y colectivos que son una necesidad de larga data.

Actualmente de no lograr cambios en la disposición del conocimiento racional y crítico de los docentes, tanto en las aulas como en la relación con la comunidad y todos los agentes que involucran el proceso educativo, se podría encontrar un sistema educativo vacío que genera ciudadanos apáticos. En este sentido un pueblo que esté acostumbrado a aislarse de la conexión entre la ciudadanía con la comunidad educativa será un pueblo marginado o dominado de pensamientos.

\section{MÉTODO}

$\mathrm{E}$ sta investigación se enmarcó en el paradigma cualitativo y por la consideración de sus objetivos fue descriptiva y bibliográfica. Se desarrolló un estudio con orientación en el área interpretativa, porque se detuvo en problemáticas humanas y se centró en aspectos individuales y cualitativos de las acciones de las personas, bajo la búsqueda de la comprensión del tema de la responsabilidad social y la ciudadanía.

Afirma, Bonilla (1997) que la investigación cualitativa intenta hacer una aproximación global de las situaciones sociales para 
explorarlas, describirlas y comprenderlas a partir de los conocimientos que tienen los diferentes actores involucradas en ellas, puesto que los individuos interactúan con los otros miembros de su contexto social compartiendo el significado y el conocimiento que tienen de sí mismos y de su realidad.

El diseño de la presente investigación cualitativa exigió que el articulista se posicionara críticamente ante concepciones de diferentes autores especialistas en el campo educativo, social y cultural.

Para la sistematización de la información, se utilizó como técnica el Análisis de Contenido, entendiéndose como la "técnica de investigación para hacer inferencias válidas y confiables de datos con respecto a su contexto" (Hernández 2003, p. 412). En este sentido se seguirá lo estipulado quienes sostienen que:

El análisis de contenido se efectuó por la codificación, que es el proceso a través de la cual las características relevantes del contenido de un mensaje son transformadas a unidades que permitan su descripción y análisis preciso. Lo importante del mensaje se convierte en algo susceptible de describir y analizar.

En este estudio se utilizó una observación participante completa, debido a que el investigador forma parte del grupo cuya característica principal es su compenetración con el grupo no se siente ajeno sino totalmente identificado. Además, su observación es libre, sin presiones, nadie se siente observado, no se sabe en qué momento está realizando su observación, no existe rechazo es un integrante más del grupo sujeto de estudio.

\section{RESULTADOS}

S e derivó de la investigación el aspecto primordial de la Responsabilidad social y formación ciudadana como elementos transformadores de la educación. Al respecto, se expuso que existe un desconocimiento casi total de los principios y valores ciudadanos, esto ha traído como resultado individuos que no reconocen su rol protagónico, el cual, al entender lo que representa verdaderamente ser ciudadanos, se tendrá como consecuencia una sociedad de respeto e igualdad que va a partir de un conocimiento de responsabilidad social producto de una memoria concientizada, crítica, dinámica, guiada por medios en este caso de centros educativos. 
Para ello se hace necesario entender bajo qué concepto se hizo referencia a lo que es ciudadanía.

Al concepto de ciudadanía le ocurre lo que describe Isabelle Stengers (2003) cuando habla de los conceptos nómadas, ya que tiene una larga historia de significados, por lo cual se tomaron los siguientes aportes: Ejercer la ciudadanía supone participar en forma activa de los asuntos de la ciudad. Se entiende la ciudad como un espacio social, cultural, económico, político, y de convivencia que se caracteriza por no ser excluyente para nadie, bajo ningún concepto. El ejercicio de la ciudadanía parte de cada persona, es todo aquello que traduce y pone en práctica vivir la ciudad como sujeto activo y responsable, es decir capas de tomas parte en lo que acontece y dar cuenta de ello. Por tanto, el ejercicio de la ciudadanía requiere sentirse concernido por los asuntos colectivos, constar y comprender que los problemas globales nos atañen. En este sentido ser ciudadano o ciudadana es atreverse a ejercer la ciudadanía, supone además sentirse capaz de tomar decisiones y actuar efectivamente.

Según Bárcena (1997) la ciudadanía es un título que sirve para reconocer la pertenencia de una persona a una comunidad política y su capacidad individual como miembro activo de esta. En este sentido, la ciudadanía equivale al reconocimiento de una serie de derechos y deberes relacionados con la participación en la esfera pública. Pero también la ciudadanía entraña un vínculo político que es proporcionar la puesta en práctica de esa clase de derechos y deberes reconocidos.

Para Bartolomé (2007) la ciudadanía implica el ejercicio activo del ciudadano/a. Desde esta perspectiva se entiende la ciudadanía como una construcción social, por tanto ser ciudadano o ciudadana no se vincula exclusivamente con la adquisición legal de un status, sino con el desarrollo de un sentimiento de pertenencia a una comunidad, que lleva a la ciudadanía a participar en los asuntos públicos y adquirir las competencias ciudadanas necesarias para tener presencia activa en el espacio público. Los tres elementos anteriores interactúan entre sí de forma dinámica, de tal manera que una fuerte dificultad o barrera en uno de ellos puede impedir el desarrollo de los demás. A su vez, el ejercicio de la ciudadanía depende del contexto social en que las personas se sitúan (familiar, escolar, social, político, etc.). 
De acuerdo con el ISO 26000, la Responsabilidad Social corresponde con la responsabilidad de una organización por los impactos de sus decisiones y actividades en la sociedad y en el medio ambiente, a través de una conducta transparente y ética que sea compatible con el desarrollo sustentable y el bienestar de la sociedad, que tome en cuenta las expectativas de los grupos de interés (en especial la de los grupos vulnerables y hacia las generaciones futuras) y que además cumpla con las normas internacionales y, a su vez, se integre por toda la organización.

Vallaeys (2006) menciona que para los británicos la responsabilidad social es considerada una herramienta de competitividad y rentabilidad, para los alemanes este concepto se encuentra centrado en la comunidad y la ciudadanía corporativa; mientras que, por ejemplo, los franceses desconfían de ella al considerarla una estrategia para manipular a la opinión pública.

Mavárez (2005) realizó un trabajo de grado titulado La Responsabilidad social como instrumento para el fortalecimiento de la vinculación Universidad-entorno social, en la Universidad del Zulia, con el objetivo de proponer lineamientos estratégicos que articulen las funciones universitarias, como lo son la docencia, investigación y extensión, con miras a fortalecer la vinculación universidad-entorno social bajo el enfoque de responsabilidad social universitaria. El trabajo fue descriptivo, analítico, de campo, con una muestra representada por el Consejo Central de Extensión de la Universidad del Zulia y sus distintas Divisiones de Extensión de las Facultades de Medicina, Ingeniería, Arquitectura, Ciencias Económicas y Sociales, Agronomía, Experimental de Ciencia y Ciencias Jurídicas y Políticas.

Para encontrar un posible camino en medio de tantas circunstancias, dicha investigación planteó un estudio del gerente como elemento transformador para las instituciones educativas en donde se desarrollaran posibles actividades que mejoren el problema de desunión y aumentara las ideas de formación ciudadana y responsabilidad social donde el docentes se relacione con el medio educativo.

Para fomentar que el docente al momento de impartir sus clases estén en la capacidad no solo de proporcionar el conocimiento intelectual sino ayudar a comprender la ciudadanía, que se realicen planes de revalorar el espacio comunitario educativo en donde participe la institución, los docentes, estudiantes, padres 
representantes, obreros, entre otros, para que cada uno conozca cual es su rol como ciudadano.

Ante semejante realidad se consideró necesario exponer la importancia de un compromiso por parte de los docentes en su labor como gerentes de proporcionar herramientas que vayan en función de la construcción del ciudadano, es indispensable plantear papeles significativos para construir una nueva educación en valores que no se quede plasmada solo en escrito sino que vaya más allá, ante la responsabilidad que se tiene frente a la sociedad para formar al ciudadano. Tal como lo afirma Pérez (2013) hoy se insiste mucho en la necesidad de educar en valores. Pero no suele decirse que hoy hace falta mucho valor para educar. Hasta hace unos años, era relativamente fácil educar. En primer lugar, había consenso entre lo que se consideraba bueno y malo y, lo que es más importante, la búsqueda y vivencia del bien parecía ser tarea de todos.

Según el Reglamento del Ejercicio de la profesión docente de la República Bolivariana de Venezuela en su capítulo I, relacionado al personal docente establece en su artículo 6 que son deberes del personal docente contribuir a la elevación del nivel ético, científico, humanístico, técnico y cultural de los miembros de la institución a la cual trabajan. De igual manera establece el cuidado y la responsabilidad con los recursos naturales y del ambiente. Por lo tanto, se hace ya evidente que la institución no representa una isla sino que se encuentra inmersa en un colectivo con características diversas y complejas.

Todos estos involucran a las instituciones educativas, por consiguiente el presente trabajo de investigación se planteó el propósito de estudiar cómo el gerente por medio de su posición y liderazgo, es el principal eje para el impulso en la formación en valores de la comunidad educativa para que estos sean ciudadanos responsables, cooperativos en el plantel, que les permitan a las instituciones unir lazos con su entorno y mejorar su capacidad institución.

\section{CONCLUSIONES}

n estas líneas finales se asevera que es necesario
profundizar más en este campo de estudio, pues está
carente en el saber que los docentes deberían ser los 
forjadores de impartir valores ciudadanos y se conozcan los roles que urgen su prevalencia en los diferentes niveles educativos, asumiendo el protagónico en la formación de futuros ciudadanos. También fomentar la creación de proyectos que brinden estrategias novedosas para el reforzamiento del sistema educativo.

Así mismo se interpretó que los principios ciudadanos sean los principales objetivos por alcanzar en la educación no solamente ciudadanos intelectuales, sino que la educación debe estar guiada a crear ciudadanos responsables socialmente bajo los valores éticos y morales, alcanzando altos estándares de calidad que permitan mejorar al individuo en su actuar diario, vinculando todo lo aprendido en su formación, ligado a su quehacer cotidiano.

\section{REFERENCIAS}

Arana, M. Batista, N. (2009). La educación en valores: una propuesta pedagógica para la formación profesional. Revista de Estudios para la Ciencia, la Tecnología y la Innovación. Valencia, España: Universidad Politécnica de Valencia

Bárcena, F. (1997). El oficio de la ciudadanía. Barcelona: Paidós

Bartolomé, M. C. (2007). Construcción de una ciudadanía intercultural y responsable. Madrid: Narcea Ediciones

Bonilla, E. Rodríguez, P. (1997) Más allá del dilema de los métodos. La Investigación en las ciencias sociales. Ediciones Unidades. Edit. Norma. Buenos Aires

Educere. Revista Española de Educación Comparada (2007). Miradas plurales sobre algunos aspectos significativos de la actual realidad educativa latinoamericana. Sociedad Española de Educación Comparada. Universidad Nacional de Educación a Distancia

Hernández. (2003). Metodologia de la investigacion. Facultad de Ciencias Económicas y Sociales. Universidad de Los Andes. Venezuela

Márquez, G. (2007). La ciudadanía como meta de la tríada republicana. Argumentos (México, DF), 20(53), 35-52

Mavárez, R. (2005). Los valores en la formación ciudadana en el estudiante de hoy. Trabajo especial de grado para optar al título de Magíster en Gerencia Educativa en la División de Estudios para Graduados de la Facultad de Humanidades y Educación de la Universidad del Zulia. Inédito 
Pérez, A. (2013). El Valor de Educar. Ediciones Centro de Formación e Investigación Padre Joaquín. Maracaibo, Venezuela: Fe y Alegría

Stengers, I. (2003). The Cosmopolitan Proposal. La Decouverte, 994-1003

Vallaeys, F. (2006). Responsabilidad Social: una urgencia filosófica. México: Iesalc 